\title{
Development of A Light Fidelity System for Improving Network Performance in a Wireless Local Area Network
}

\author{
S.N. Arinze, G.N. Onoh and D.O. Abonyi \\ Corresponding Author: S. N Arinze
}

\begin{abstract}
Network performance is the measure of the quality of service offered by the network. It hinders when multiple loads are running on a wireless local area network, thereby causing slow down in social communication, networking, and business activities. This paper aimed at improving network performance in a wireless local area network using light fidelity technology. The light fidelity system designed and developed was a bidirectional system of downlink and uplink transceiver circuits. Physical measurement was carried out on the developed light fidelity system using Netlimiter software to capture the network performance. Results obtained showed that the performance of light fidelity systems throughput was high, while the latency and packet loss was low. The network performance of the light fidelity system was better than the wireless fidelity technology in the wireless local area network. Its network performance is not significantly affected by the number of users and hence should be utilized in a high-density environment.
\end{abstract}

Index Terms - Light Fidelity, Wireless Fidelity, Throughput, Latency, Packet loss, Netlimiter Software.

\section{INTRODUCTION}

Today, radio frequencies have brought internet or wireless data communication to the world. Messages can now transmit at short distances as well as ranges of thousands or millions of kilometers. The radio frequency spectrum is a significant source of revenue in Nigeria's economy as a result of its direct application in telecommunication, military operation, social services, and scientific research. Many industries depend heavily on it. By 2016, five billion people had access to the internet, according to an estimate by the International Telecommunications Union (ITU) [1]. This tremendous increase in the demand for wireless data has made the available radio frequency bandwidth filled up and slow in operation. The slow response experienced by users while accessing the wireless network motivated this research. The slow speed of the network leads to long processing hours especially when many users connect to the system. Sometimes pages will not load, emails will buffer and email crawls to a halt and some even get a dreaded error message that the webpage is not available. Hence the wireless network in Nigeria cannot provide fast and reliable data transmission due to congestion. Deploying more cell towers does not double the capacity. In the desire to control and manage the available radio frequency, the Nigerian Communications Commission (NCC) has introduced the following management techniques: spectrum planning, frequency assignment, spectrum refarming, and monitory policy. However, the

S.N. Arinze, G.N. Onoh and D.O. Abonyi are with Electrical and Electronic Engineering Department, Enugu State University of Science

and Technology ～(email:arinze.ndidi@esut.edu.ng, greg.nwachukwu@esut.ng.edu, abonyi.dorathy@esut.ng.edu). 
resource is scarce and hence, cannot cope or overcome the rapidly growing wireless data traffic [2].

Radio waves seem to have limited bandwidth and speed because they have been fully exploited and congested. This congestion has paved the way for the need for spectral relief and larger bandwidth. Other spectrums need exploration. The simplest solution is an improved bandwidth. Based on this scenario, one could ask if the world can use another frequency to complement the existing radio frequencies in accessing the internet. A possible approach and adequate answer is the Visible light spectrum. It communicates at a very high speed and has a natural source of energy. It has frequencies between $400 \mathrm{THz}$ to $800 \mathrm{THz}$, and it's ten thousand times more compared with radiofrequency ( $3 \mathrm{KHz}$ to $300 \mathrm{GHz}$ ). Visible light is safe since it reflects, and our bodies are not transparent to it, unlike the radiofrequency radiation that is detrimental to the environment and human health. The light spectrum is presently unlicensed, has wide bandwidth, and can exploit with existing infrastructures. Hass coined the term light fidelity (Li-Fi) at the Technology, Entertainment, and Design (TED) Global talk, where he brought in the idea of wireless data from every light bulb [3]. He streamed a video of a blooming flower using a table lamp with a Light-emitting diode (LED) bulb. He went further to block the light from the bulb, which showed that the light-emitting diode was the source of video data. Hass called it a new generation of high brightness where you transmit a digital ' 1 ' if the LED is ON and transmission of ' 0 ' if it is OFF. Fourteen billion light bulbs [4] exist in this world, and if replaced with the LED ones that transmit data, it means that lightning bulbs can bring not only the light but wireless connection at the same time. Every object can be a light source once it illuminates. Examples are bulbs, television, and screen of mobile phones.

Several authors [5] proposed the implementation of a simple Li-Fi based system where data transfer is from one device to another. Data was encrypted, converted into binary form, and sent to the transmitter module, which generates the corresponding on-off pattern of the LEDs. The receiver module, which has a photodiode to detect the on and off states of the LED, generates the binary sequence, and converts the data to the original format. The data transfer speed was low. It can improve by using white LEDs that have high-frequency speed and brightness. The two-stage access point of selection for hybrid visible light communication (VLC) and radio frequency(RF) networks using fuzzy logic carried out was by considering different required data rates; some users attached to the VLC and the remaining ones assigned to RF [6]. Integrated Li-Fi smart communication through illumination presented by authors [7] was on data transmission between two devices. Data was modulated and sent to LEDs on

the transmitter part while photodetector receives it, demodulates and decodes it. Several authors [8] worked on Visible light communications using a blue GaN $\mu$ LED and fluorescent polymer color converter. The technique used was the DC-biased optical orthogonal frequency- division multiplexing (OFDM). Incoming bitstream was encoded and turned into a signal. DC bias added to the message was because of the broad peak to average power ratio of OFDM. The modulated white light generated from LED focused onto an avalanche photodetector. In this work, a developed light fidelity system improved the performance of wireless local area networks.

\section{THEORY}

Light Fidelity ( $\mathrm{Li}-\mathrm{Fi})$ is a technique that uses light as a means of communication. It falls under the category of the visible light spectrum having a wavelength of $780 \mathrm{~nm}-375 \mathrm{~nm}$ and frequency of $480 \mathrm{THz}-800 \mathrm{THz}$ which is unregulated, unused and can communicate at very high speeds. Li-Fi is a technology that transmits data using a light-emitting diode (LED) as a transmitter while the photodiode acts as the receiver and convert the light energy into electrical signals [9]. Li-Fi's objective is to increase data rates in wireless communication. It 
transmits data by applying a constant current to the LED, but the output can vary at high speeds by fast and subtle variations of the electric charge. It can be achieved by quickly switching on and off of the LED. A digital ' 1 ' is transmitted if the LED is on and digital ' 0 ' if the LED is off. The intensity variation happens in such a way that the human eye cannot detect it. The light fidelity system converts the input electrical signal to optical energy by transforming the data input from the internet into byte format and transmitted in the form of flashes of LED which are received by the photodiode. A photodiode is a photodetector that captures the sequence and converts it into electrical signals which are amplified, transformed into the original format and presented as output in the laptop.

Li-Fi main components are :

(a) Light Emitting Diode (LED): This is a p-n junction diode that emits light when current flows through it. Its made up of direct semiconductor materials such as Gallium arsenide, Aluminium gallium indium phosphide, Indium gallium nitride, etc.) and converts electrical energy into light. The advantages of LEDs are long life and robustness as it can withstand external shock, slow failure. It radiates very little heat, lights up very quickly, has low power consumption, low cost, and small in size [10].

(b) Photodiode: It is a p-n junction or pin semiconductor device that converts light energy into electrical energy. It is very sensitive to light. When light strikes on it, the valence electrons in the depletion gains enough power and moves freely from one region to another carrying electric current, gets attracted towards the positive terminal of the battery and thereby generates photocurrent. Quick response time, spectral sensitivity, and sizeable radiant sensitivity area are the essential requirements of a photodiode. The advantages of Photodiode are low noise, high gain, high response speed, high sensitivity to light, low sensitivity to temperature, low cost, small size, and long lifetime [11]. Photodiodes operate as photodetectors, tomography, smoke detectors, and blood gas monitors.

Light Fidelity is safe and used in areas where RF communication cannot fit in, such as aircraft, underwater, and hospital without causing interference [7]. In aviation, it cannot interfere with the pilot's navigation systems and as well provide high-speed internet using the overhead bulb present in the airplane. Even in underwater applications, it could replace wires used in underwater remotely operated vehicles with a high powered lamp or headlamps for communication and data processing. Li-Fi can be used to control the medical equipment and avoid any of it being hazardous to the patient's health [12]. Li-Fi cannot penetrate the walls because of its shorter wavelength, but it is a potential candidate for mobile connectivity yielding higher data rates and higher security.

\section{MATERIALS AND METHOD}

Bidirectional communication for the Li-Fi system involves uplink and downlink transceiver circuits. In the design of the downlink Li-Fi transceiver circuit, the characteristic impedance of LAN output taken as $100 \mathrm{ohms}$ is in line with the standard norm of IEEE. At the transmitter side, 28 pins ML4664 single-chip integrated circuit LED driver shown in fig. 1 was used to obtain superior performance and avoid system complexity. ML4664 is an LED driver that converts 10BASE-T Copper media to 10BASE -F Fiber media and offers a current output that directly drives an LED. 


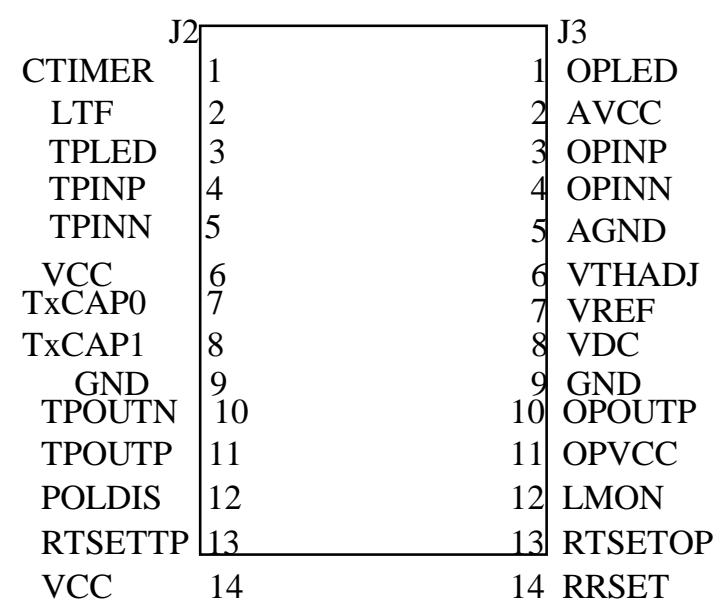

Fig.1 ML4664 LED driver

ML4664 complies with the characteristic impedance of LAN output or drives $100 \mathrm{ohms}$ unshielded twisted pair. Before the data goes to the differential inputs (TPINP \&TPINN) of the ML4664, an RC low pass filter was connected to the RJ45 ethernet cable to pass lowfrequency signals, filter out noise, have excellent frequency response and as well for the integrated circuits reliability and low power consumption. The RC circuit was implemented by having $50 \mathrm{ohms}$ resistor connected in each of the differential inputs and a combination of it $(\mathrm{R}=100 \mathrm{ohms})$ in series with a decoupling capacitor $(\mathrm{C}=0.1 \mathrm{microfarad})$ as shown in fig. 2

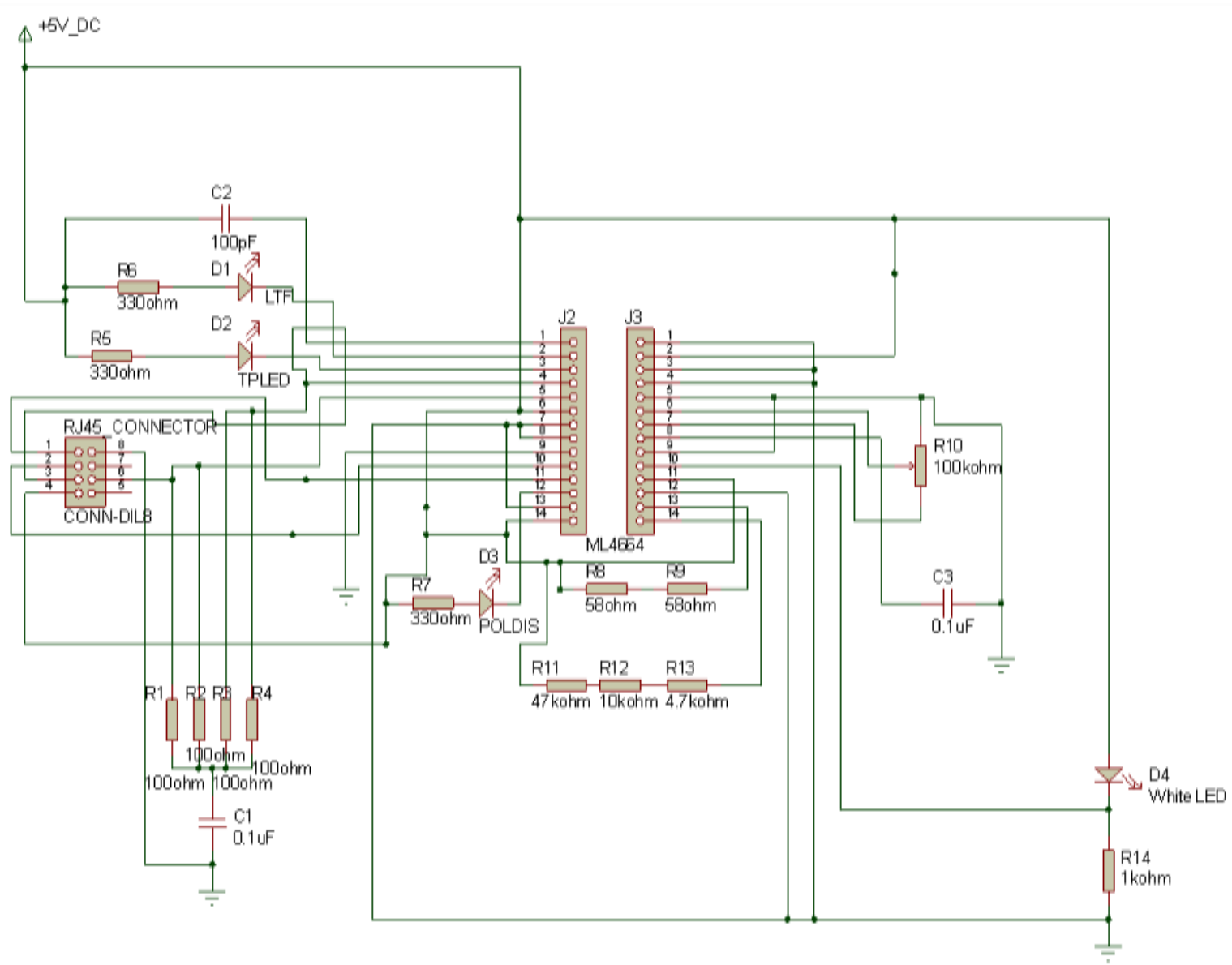

Fig.2 Designed Li-Fi Downlink Transmitter Circuit 
The differential inputs twisted-pair TPINP(pin 4 of J2) and TPINN(pin 5 of J2) of ML4664 received the data, buffer and send it to the LED driver pins to modulate the intensity of the LED. The driver pins are:

- LTF (Pin 2 of J2) which indicated that the link was working.

- TPLED (Pin 3 of $\mathbf{J} 2$ ) which showed that the reception was occurring.

- POLDIS (Pin 12 of $\mathrm{J} 2$ ), which received the status of the polarity.

These pins connect with each LED to show their working or communicating status by connecting a determined $330 \Omega$ resistor between each LED and the voltage supply. The value of the resistor calculated was by using the values of forward-voltage $V_{f} \rightarrow 1.8 \mathrm{v}$, power supply voltage $V_{C C} \rightarrow 5 v$, current on LED $I_{L} \rightarrow 10 \mathrm{~mA}$ from Micro Linear datasheet.

Recall that ;

$$
V_{C C}-V_{f}=I_{L} R
$$

Substituting the values obtained from the datasheet in equation $(1)$ gave $\mathrm{R} \approx 330 \Omega$.

The LED driver output, which is OPOUTP (Pin 19 of J3), controlled the amount of current going to the white LED through a resistor of $1 \mathrm{~K} \Omega$. The value of the resistor was by substituting the values of $V_{f} \rightarrow 1.8 v, V_{C C} \rightarrow 5 v, I_{L} \rightarrow 3.2 \mathrm{~mA}$ obtained from the Luxeon Rebel datasheet into equation (1).

A resistor of $115 \Omega$ connects between the pins of RTSETOP (Pin 13 of J3) and $V_{C C}$ (Pin 14 of J2) to set the current driven output going into the white LED and a $61.9 \mathrm{k} \Omega$ resistor bound between the pins RRSET(Pin 14 of $\mathrm{J} 3$ and $V_{C C}$ (Pin 14 of $\mathrm{J} 2$ ) For internal biasing. Also, pins VRef (Pin 7 of J3) and VTHADJ( Pin 6 of J3) were connected with 100k $\Omega$ to increase the sensitivity of ML4664. All the GND pins were grounded while $V_{C C}$ Pins connect to 5v for the proper functionality of the integrated circuit.

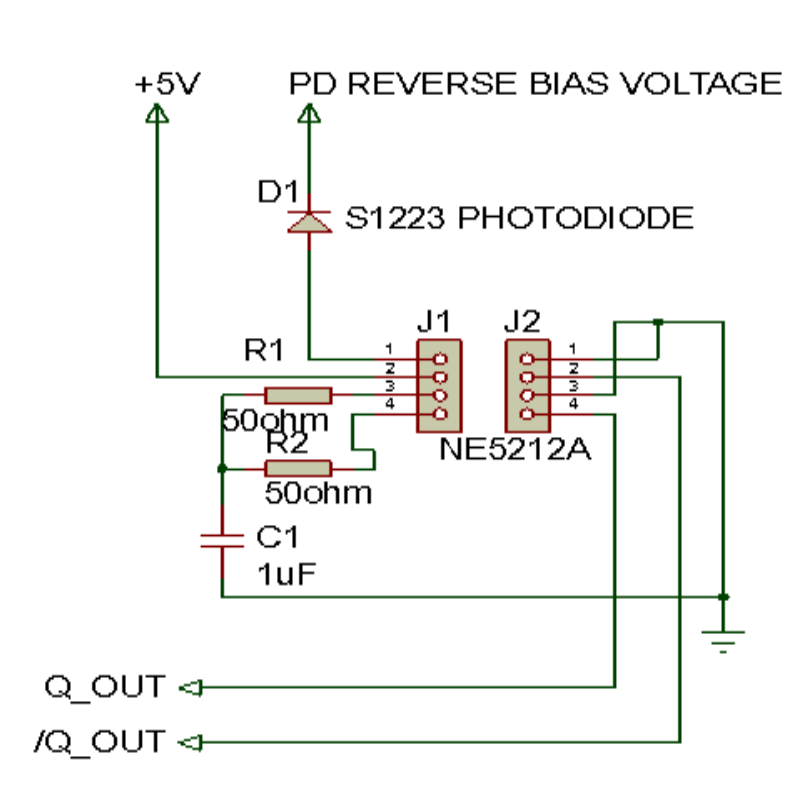

Fig. 3 The downlink receiver circuit of light fidelity

At the receiver section, $12 \mathrm{v}$ power supply with voltage doubler as shown in fig. 3 connects to the S1223 Si PIN photodiode. The S1223 photodiode connects to the input (Pin 1 of J1 ) of NE5212A $140 \mathrm{MHz}$ transimpedance amplifier which amplified and converted the current from photodiode to a differential voltage output in Pin 4 of J2(OUT +) and Pin 2 of J2(OUT-) 
which contains both analog and digital signal. Pin 2 of $\mathrm{J} 1\left(V_{C C}\right)$ was connected to $5 v$ to supply power to the transimpedance amplifier while Pin 1 of $\mathbf{J} 2\left(G N D_{1}\right)$ and Pin 3 of $\mathbf{J} 2$ $\left(G N D_{2}\right)$ were connected to the ground as required for the proper function of the transimpedance amplifier (Philips, 2015). The transimpedance amplifier has an internal limiting amplifier that takes only the digital signals from the output voltage. It also acts as a differential line amplifier by driving the $100 \Omega$ terminated cable through the connection of a $50 \Omega$ resistor in series with a $1 \mu \mathrm{f}$ capacitor via Pin 3 of $\mathrm{J} 1$ and pin 4 of $\mathrm{J} 1$. After that, it pushes the data through Pin 4 of J2(OUT +) and Pin 2 of J2 (OUT-) over the RJ45 Ethernet cable to the laptop.

In the design of the uplink Li-Fi transceiver, the ethernet cable from the laptop was connected to the NE5212A transimpedance amplifier through a $100 \Omega$ resistor for the avoidance of impedance matching of the LAN output. It enables the pulses to have non-distortion. Since the Transimpedance amplifier has differential input, two equal coupling capacitors of 0.01 microfarad was connected to achieve a high pass filter. It was to filter out DC signals before it was linked to the inputs of the LED driver, as shown in fig.4.

An external $0.1 \mu \mathrm{f}$ capacitor nulls the offset and error signal of the transimpedance amplifier. The inputs OPINN (Pin 3 of J3)and OPINP (Pin 4 of J3) of the ML4664 LED driver received the data and sent it to the driver's pins which are:

- OPLED(Pin 1of J3) which showed that reception with the input pins are working.

- LMON(Pin 12 of J3) which indicated that data is processed.

LEDs were connected to each of the driver's pins to display their working status. by attaching a $330 \Omega$ resistor between the LEDs and $V_{C C}$.

The LED driver output, which is OPOUTP (Pin 10 of J3), was connected to the Infrared LED that transmitted the data through a resistor of $330 \Omega$. Infrared LED used was because of the wavelength separation between it and the white LED. Thus it will not interfere with bidirectional communication.

Three $220 \mathrm{pF}$ in parallel, bound between pin TxCAP0 and TxCAP1A, was for preequalization and reverse of any distortion in the twisted cable. The pins VRef (pin 7 J3) and VTHADJ ( pin $6 \mathrm{~J} 3$ ) connects with 100k $\Omega$. All the GND pins were grounded while VCC connects to the $5 \mathrm{v}$ supply.

The uplink receiver section is the same as the downlink receiver, as shown in fig.3. The downlink and uplink transceiver circuit was developed on the stripboard to form a prototype and integrated to access the performance of the light fidelity system, as shown in fig.5. The set up of the transmitter and receiver section was in such a way that both were facing each other to ensure meaningful communication. Setup of Internet connection occurred, and a download of the data size of 29.12 MB was from the Youtube website through Mozilla Firefox application for one laptop device. NetLimiter software installed on the laptop measured the traffic statistics.

In the Wireless Local area network, which utilizes wireless fidelity (Wi-Fi) technology, a fourth-generation $(4 \mathrm{G})$ modem connects to the LB-LINK Wi-Fi router through the USB port. The LB-LINK Wi-Fi router LAN port was connected to the LAPTOP using an RJ45 network cable. It was configured by connecting to its portal on the Mozilla web browser using its IP address. The $4 \mathrm{G}$ parameter set up was a means of access to the router. The operation mode set as a gateway and the access point name, username, and password was a means of gaining a connection to the internet. The security key was enabled to have control of the connected users. The NetLimiter software was installed on the laptops to capture the traffic statistics and analyze the network performance when downloading data of 29.12MB over the internet. 


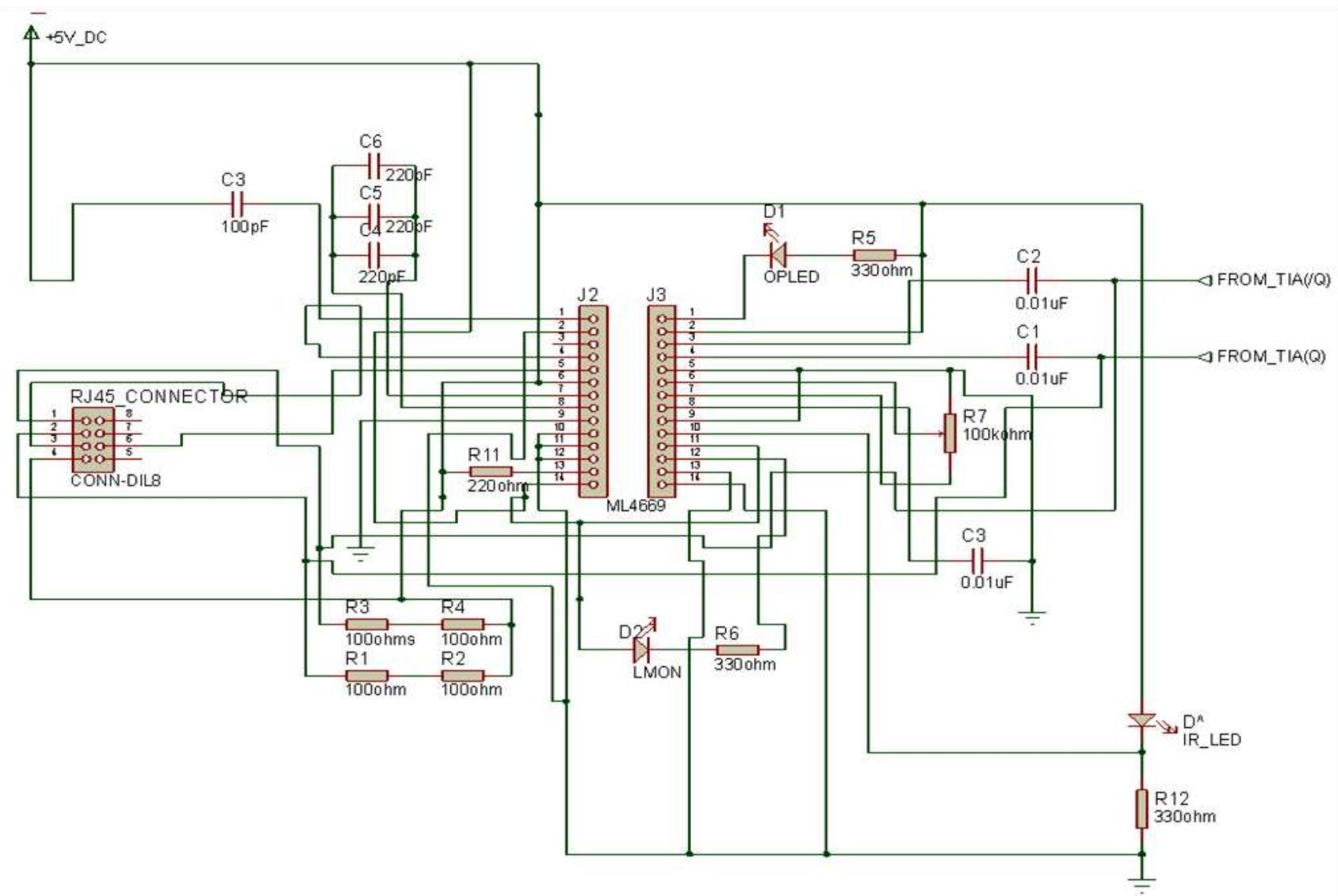

Fig. 4 Light fidelity uplink transmitter

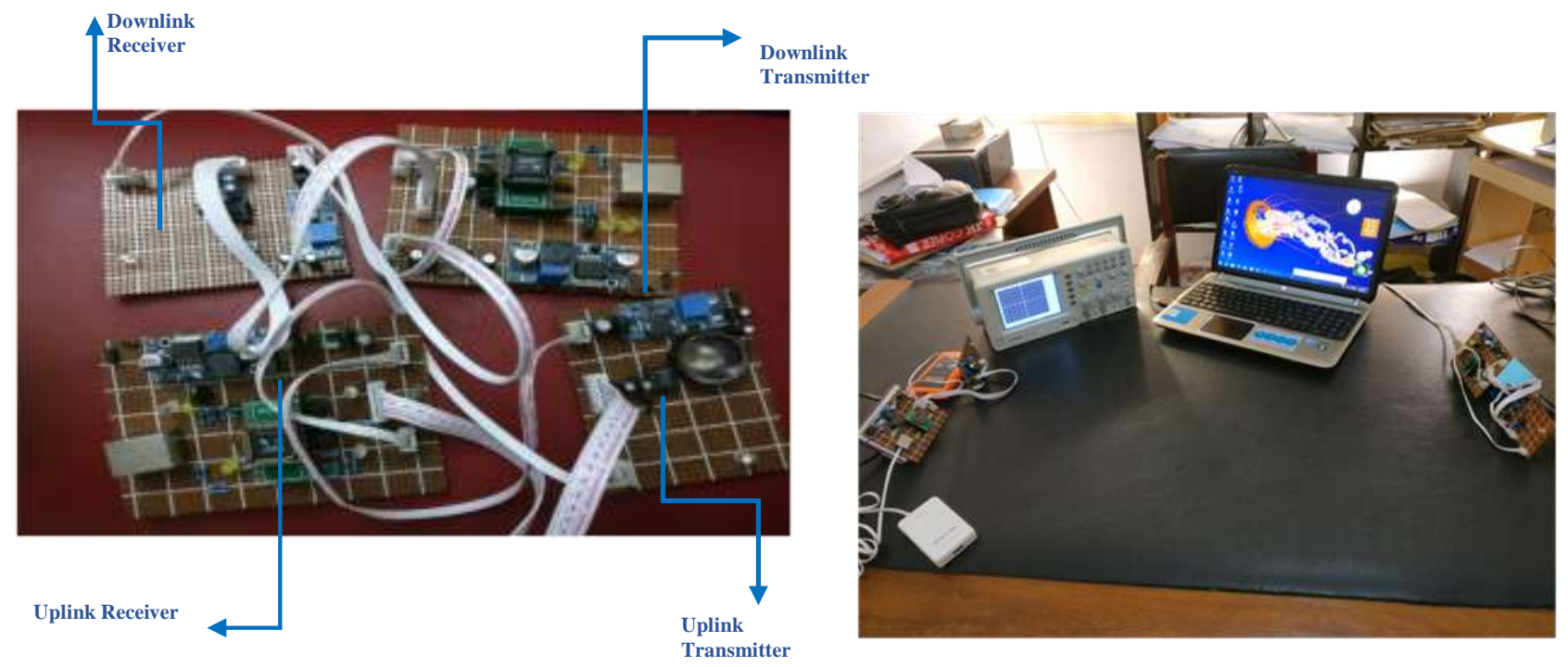

Fig.5 Developed light fidelity network 


\section{RESULTS}

The developed Li-Fi system using a network interface card was connected to the internet at Enugu State University of Science and Technology EXXON MOBIL Laboratory for ten users. Data of $29.12 \mathrm{MB}$ was downloaded from the YouTube website using the Mozilla Firefox application browser, and the NetLimiter software captured the traffic statistics. The result in fig.6 presents the network performance of the Li-Fi system and the Wi-Fi system.

The result in fig. 6 showed that the throughput decreases as more users connect to the Wi-Fi System. It was because of the sharing of the available bandwidth between the users. It has an exponential decay. Between the fourth user and tenth user, the decrease tapers. The tenth user was tending to zero. Thus by extrapolation, it can be seen that connecting more than users will result in zero output. The addition of more users exceeds the capacity of the network. Each additional user decreases the value obtained by every other user. The amount of simultaneous data injected into the system resulted in transmission delay, packet collision, retransmission of data, lowers the throughput, and thereby deteriorates the performance and quality of the network.

The figure also showed that the latency had exponential growth. It was as a result of an overloaded Wi-Fi router /modem. The router will eventually slow down when too many clients are using it at the same time. The clients were waiting for each other's requests to be processed, thereby causing lag. Also, spikes in internet utilization during peak usage often caused the slowdown. The shared internet Wi-Fi server by the users became overloaded with activity. Simultaneous downloads and retransmission occurred and thereby causes delay or slow down of the network. Increasing the number of users will increase the transmission time delay of all packet sizes. Packet loss increased from the first to the third user and maintained a constant value. After the ninth user, it rose exponentially. The packet loss was as a result of the increased number of users in the network. As the number of users increases, more packets pushed into the network were waiting in the MAC layer for the procession. When the packets exceeded their waiting time limit, they were discarded by the MAC layer though they are retransmitted, which depends on the user. The points where the packet loss was zero have high latency. As more users are downloading data, many packets are lost.

The throughput of the Li-Fi network was high and kept increasing linearly as more users connect to it. It was because that light has a wide bandwidth. There is low latency in the Li-Fi network, and it decreases because Li-Fi transfers data at high speed with little or no retransmission. The packet loss obtained was lesser in the Li-Fi system and did not change because there was no congestion.

The network performance of the light fidelity system was better than the wireless fidelity system because the bandwidth is not constrained. Li-Fi system gave high throughput, low latency, and low packet loss, and the number of users is not significantly affected in the Li-Fi system. It makes $\mathrm{Li}-\mathrm{Fi}$, an ideal system for data transmission in a highly dense area. 

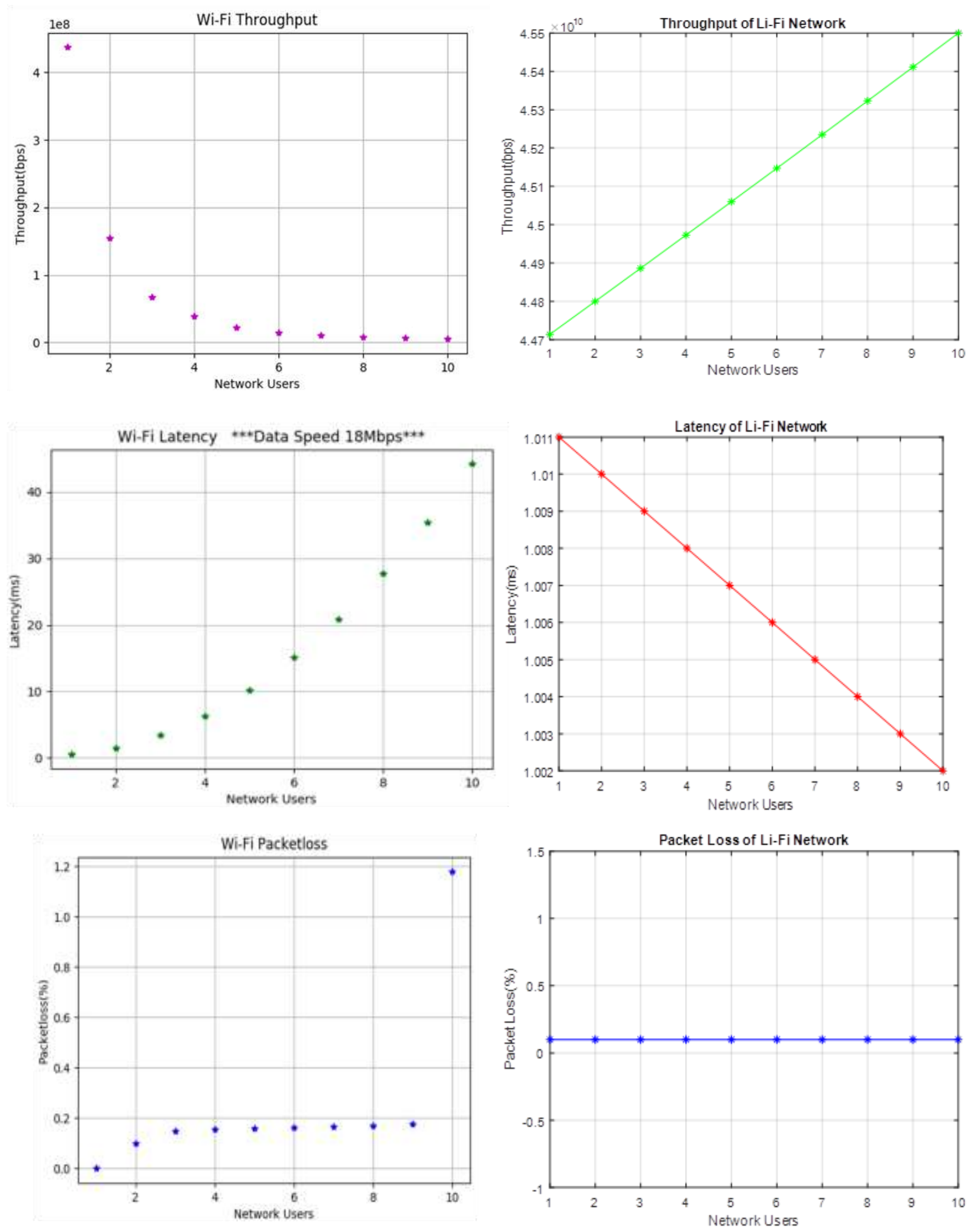

Fig. 6 Network performance of wireless fidelity and light fidelity system 


\section{CONCLUSION}

The need and demand to be connected to the internet and experience an excellent performance of a wireless local area network has led to the improvement of the Wireless Fidelity (Wi-Fi) technology and creation of Light Fidelity (Li-Fi) System. It uses part of the electromagnetic spectrum that is not much utilized. The deployment of Li-Fi technology can solve the problem of lack of radio spectrum and low data throughput in internet surfing. Although this Li-Fi technology has not made a commercial success in the market, it is a significant potential in the field of wireless internet that can complement the existing wireless fidelity system in obtaining a high network performance. Light fidelity is a growing technology that can take communication to a new era.

\section{REFERENCES}

[1] Rutkowski, R. (2017). RF Engineers are bringing the Internet to the World. https://www.google.com/amp/blog.bliley.com/rf-engineers-bringing-the-internet-to-theworld\%3Fhs-amp\%3Dtrue. Accessed on December 5, 2017.

[2] Nigerian Communications Commission, (2018). Frequency Management Policy. https://www.ncc.gov.ng/technology/spectrum/frequency-management. Accessed on May 24, 2018.

[3] Hass H., et al. (2016). What is Li-Fi?. Journal of Lightwave Technology, 34(6), 15331544.

[4] Haas H. (2016). Li-Fi: Conceptions, Misconceptions, and Opportunities. IEEE Photonics Conference, 680-681.

[5] Rahul, S., Akshay, S., Sandhya, P.(2014) Implementation of a simple Li-Fi based System. International Journal of Computing and Technology, 1(9), 35-39.

[6] Xiping, W., Dushyanta, B., Majid, S. (2016). Two-stage Access point Selection for Hybrid VLC and RF Networks. IEEE 27th Annual International Symposium on Personal, Indoor and Mobile Radio

Communications (PIMRC), Mobile and Wireless Networks, 19( 5), 21-27.

[7] Mahendran, R. (2016). Integrated LiFi (Light Fidelity) for Smart Communication through Illumination. IEEE International Conference on Advanced Communication Control and Computing Technologies (ICACCCCT), 53-56.

[8] Hyunchae. C. et al. (2014). VLC Communication Using a Blue GaN $\mu$ LED and Fluorescent Polymer Color Converter. IEEE Photonics Technology Letters, 26(20), 20352038.

[9] Prateek, G., Aditya, S., Prashant, K. (2016). Various Modulation Techniques for Li-Fi. Smart and Innovative Technologies in Engineering and Sciences, 5(3), 121-125.

[10] Electronics Notes, (2017). Light Emitting Diode. https://www.electronicsnotes.com/articles/electronic_components/diode/light-emitting-diode-led-technology-howdoes-led-work.php. Accessed on May 25, 2018. 
[11] Gupta Himanshi, (2016). Photodiode Modes of Operation, Working principle, and Applications. https://analyseameter.com/2016/03/photodiode-operation-modes-workingapplications.html.Accessed on May 20, 2018.

[12] Abhishek, P., Akash, A., Arpit, B., Indrago, K. (2015) LiFi: Data Transmission through Illumination. International Journal of Scientific and Engineering Research, 6(10), 14321436.

[13] Micro Linear Corporation, (2000). ML4664/ML4669 Data Sheet. https://www.alldatasheet.com/datasheet-pdf/pdf/75741/MICRO-LINEAR/ML4664.html. Accessed on June 30, 2018.

[14] Stephen Graber, (2016). 10Mb/s Single Twisted Pair Ethernet: Preliminary Cable Properties.

http://www.ieee802.org/3/10SPE/public/Sept2016_Interim/Graber_10SPE_02a_0916.pdf. Accessed on March 18, 2018.

[15] Lumileds, (2016). LUXEON Rebel General Purpose Product Datasheet. https://www.liumileds.com/uploads/28/DS64-pdf. Accessed on May 14, 2018. 